\title{
APPLICATION OF COMPRESSED SAMPLING FOR SPECTRUM SENSING AND CHANNEL ESTIMATION IN WIDEBAND COGNITIVE RADIO NETWORKS
}

\author{
Naveen Kumar and Neetu Sood \\ Department of Electronics \& Communication, Dr B R Ambedkar NIT Jalandhar, India
}

\begin{abstract}
In the last few years Compressed Sampling (CS) has been well used in the area of signal processing and image compression. Recently, CS has been earning a great interest in the area of wireless communication networks. CS exploits the sparsity of the signal processed for digital acquisition to reduce the number of measurement, which leads to reductions in the size, power consumption, processing time and processing cost. This article presents application of CS for the spectrum sensing and channel estimation in Cognitive Radio (CR) networks. Basic approach of CS is introduced first, and then scheme for spectrum sensing and channel estimation for CR is discussed. First, fast and efficient compressed spectrum sensing (CSS) scheme is proposed to detect wideband spectrum, where samples are taken at sub-Nyquist rate and signal acquisition is terminated automatically once the samples are sufficient for the best spectral recovery and then, after the spectrum sensing, in the second phase notion of multipath sparsity is formalized and a novel approach based on Orthogonal Matching Pursuit (OMP) is discussed to estimate sparse multipath channels for CR networks. The effectiveness of the proposed scheme is demonstrated through comparisons with the existing conventional spectrum sensing and channel estimation methods.
\end{abstract}

\section{KEYWORDS}

Compressed Sampling; Cognitive Radio; Spectrum Sensing; Channel Estimation; Orthogonal Frequency Division Multiplexing.

\section{INTRODUCTION}

According to Kotelnikov, Nyquist and Shannon [1-3], a continuous-time band-limited signal $x(t)$ with bandwidth $B>0$, can be exactly recovered from twice as many samples per second as the highest frequency present in the signal i.e. $2 \mathrm{~B}$, also known as the Nyquist rate. Around 2004, David Donoho, Terence Tao and Emmanuel Candès [4-6] proved that if the signal is sparse in one domain, the signal may be reconstructed back with even fewer samples than required by Nyquist's sampling theorem. In this way the sampling of the signal at sub-Nyquist rate is the basis of compressed sampling.

There are two primary conditions for the perfect recovery of the signal [7]. The first one is sparsity i.e. the non-zero terms, of the signal. CS approach is based on the fact that the most of the signals can be well-approximated as sparse signals by expanding them in a suitable sparsifying basis. The second condition is incoherence, discussed in detain in Section 2, which is applied through the restricted isometric property for sparse signals [8]. 
In the field of wireless communication system, there are many cases, where a large amount of information should be processed to obtain the interior characteristics, such as signal detection, channel estimation, network monitoring and data fusion in Cognitive radio (CR) networks. These problems share a common feature that the information being processed is huge but sparse in nature. For such cases, CS can be used to lower all of the cost attributes and increase efficiency.

Fast and accurate spectrum sensing is extremely important in wide band CR networks. To implement wideband spectrum sensing, CR needs fast Analog-to-Digital Converter (ADC) but the achievable sampling rate of ADC is only 3.6 Gsps. Capitalizing on the wideband signal spectrum sparseness, CS technique can be employed in spectrum sensing [9] in CR network.

In most scenarios in CR networks, the number of used channels is comparatively much lesser than total channels; those are vacant at a particular time and space. Therefore, when dealing with channel estimation problem in CR system where the channel band is really wide and dynamic and occupation information of the channels is compressible, CS can be exploited, since CS does not require any knowledge of the underlying multipath channel, based on the fact that a sparse structure is exhibited by the physical multipath channels in angle delay Doppler spreading, especially at large signal space dimensions, it is advantageous to utilize sparse channel estimation method based on convex/linear programming, which can be proved to outperform the existing least square based methods [10]. Such channels with significant angle delay Doppler spreading can be handled by CS algorithms in the form of Basis Pursuit (BP) and Orthogonal Matching Pursuit (OMP), when coupled with a channel equalizer mitigating inter-carrier interference, as demonstrated in [11] using numerical simulation and experiments.

The remainder of this paper is structured as follows- Section 2 presents notations. Section 3 summarises the related works, In Section 4, the fundamentals of CS theory are introduced, and the reconstruction methods of CS are summarized. Then for spectrum sensing and channel estimation, CR system model is presented in section 5. Section 6 explains the theory of different components of CS algorithms. CS based fast and efficient spectrum sensing scheme is presented, after that in section 7 we formalize the notion of sparse multipath channels to apply the compressed channel estimation based on various CS algorithms. Section 8 demonstrates and summarizes the performance advantages of proposed CS based scheme over traditional energy detection spectrum sensing and maximum likelihood ratio based channel estimation techniques. Finally, we conclude the article in Section 9 by discussing some of the finer technical details pertaining to the results presented in the paper and future scope.

\section{NOTATIONS}

$\begin{array}{ll}\mathrm{X} & \text { Discrete time signal } \\ \Psi & \text { Sparsifying basis } \\ \mathrm{S} & \text { Non zero coefficients } \\ \phi & \text { Measurement matrix } \\ \mathrm{m} & \text { Number of measurements } \\ \mathrm{Z} & \text { Additive white Gaussian noise } \\ \mathrm{R}_{\mathrm{i}} & \text { Training subset } \\ \mathrm{T}_{\mathrm{i}} & \text { Testing subset } \\ \mathrm{V}_{\mathrm{i}} & \text { Verification parameter } \\ \mathrm{C} & \text { Constant } \\ \mathrm{K} & \text { Signal level } \\ \mathrm{T}_{\mathrm{s}} & \text { OFDM symbol duration } \\ \mathrm{T}_{\mathrm{G}} & \text { Guard interval }\end{array}$




\section{RELATED WORK}

To implement wideband spectrum sensing, CR systems need some essential components, i.e. wideband antenna, wideband radio frequency front-end, and high speed Analog-to-Digital Converter (ADC). [12] and [13] developed the wideband antenna and the wideband filter efficiently but the development of ADC converter is relatively behind as the ADC currently have limited sampling rates, of the order of $1 \mathrm{GHz}$, which is not sufficient for wideband spectrum.

To solve this problem, Z. Tian and Giannakis [14] applied Compressive spectrum sensing (CSS) theory to CR systems for acquiring wideband signals at sub-Nyquist sampling rates. Capitalizing on the sparseness of the signal spectrum in open-access networks, compressed sensing techniques were developed for the coarse sensing task of unutilized spectrum i.e. spectrum holes' (SHs) identification.

Additionally, Malioutov et al. [15] described a stopping rule for a desired tolerance in reconstruction for the near-sparse case. Simin Bokharaiee et al. [16] applied cyclic prefix correlation coefficient (CPCC) based blind spectrum sensing for orthogonal frequency division multiplexing based cognitive radio (OFDM-CR) systems. Furthermore, by considering multipath correlation in the generalized likelihood ratio test (GLRT) algorithm and employing the inherent structure of OFDM systems a low complexity algorithm was obtained. Furthermore, M. Farrag et al. [17], proposed Distributed Sensing Matrix (DSM) based algorithm to reduce complexity at each sensing node and improve the unlicensed users i.e. primary users' (PUs) detection performance.

After spectrum sensing, deactivated subcarriers in non contiguous OFDM complicate the design of efficient pilot symbols for channel estimation [18-21]. In [22], the pilot design is formulated as an optimization problem minimizing an upper bound related to the Mean Square Error (MSE), where the pilot indexes are obtained by solving a series of 1-dimentional low complexity problems. To reduce the computational complexity for the optimal selection of pilot subcarriers, cross entropy optimization was utilized in [24] to determine the position of pilot subcarriers.

Manasseh et al. [23], proposed a cross-entropy optimization based pilot design scheme using convex optimization together to minimize the MSE. Min Jia et al. [25] presented channel estimation algorithm for OFDM-CR, based on OMP and applied sparsity adaptive matching pursuit (SAMP) algorithm for the first time for channel estimation in NC-OFDM systems. Moreover, for the reconstruction time-consuming of SAMP algorithm was too large, modified adaptive matching pursuit (MAMP) algorithm was introduced as an improved SAMP algorithm. Chenhao Qi et al. [26] introduced sparse channel estimation (SCE) scheme in OFDM-CR, where pilot design was formulated as an optimal column selection problem and constrained cross entropy optimization based scheme was proposed to obtain an optimized pilot pattern.

\section{COMPRESSED SAMPLING THEORY}

According to the theory of CS, information about certain signals can be acquired directly and recovered back with high probability from far fewer measurements than traditional methods, provided that the signal is sparse, by solving an optimization problem with the small amount of projections. Consider a discrete time signal $\mathrm{x} \in \mathcal{P}^{\mathrm{N}}$, which can be expressed as $\mathrm{X}(\mathrm{n})$ where $\mathrm{n}=1$, $2, \ldots, \mathrm{N}$. The claim of compressive sensing is that from $\mathrm{m}(\mathrm{m}<<\mathrm{n})$ measurements, we can reconstruct the original signal $\mathrm{x}$ with non-adaptive linear measurements. This does not violate the Shannon-Nyquist sampling theorem as reconstruction of only sparse signals is possible. 


\subsection{SPARSE REPRESENTATION OF THE SIGNAL}

Based on the CS theory, efficiency of signal acquisition depends on the sparse representation of the signal. Signal $X \in \mathcal{P}^{N}$ can be expressed in a sparsifying basis $\Psi=\left[\Psi_{1}, \Psi_{2}, \ldots \Psi_{N}\right]$ as follows [27] :

$$
\mathrm{X}=\sum a_{N} \psi_{N}=\psi a
$$

where $a$ is the vector of inner products, $a_{\mathrm{N}}=\left\langle\mathrm{X}, \psi_{\mathrm{N}}\right\rangle$. When only $\mathrm{S}(\mathrm{S}<<\mathrm{N})$ of the $a_{\mathrm{N}}$ coefficients are nonzero, it is called S-sparse.

\subsection{MEASUREMENT MATRIX}

By using non-adaptive linear projections the useful information in the sparse signals can be acquired, via directly compressing signals into a smaller data [27]. Measurement of the signal $\mathrm{x}$ is done by sampling it with respect to a measurement matrix $\phi \in \mathcal{P}^{\mathrm{M} \times \mathrm{N}}$. Substituting $X=\psi a$ into Equation (1):

$$
\mathrm{Y}=\phi \mathrm{X}=\phi \psi a=\Theta a
$$

where $\mathrm{Y}$ and $\phi$ are $\mathrm{M} \times 1$ column vector and $\mathrm{M} \times \mathrm{N}$ measurement matrix respectively. Based on the CS theory signal recovery can actually be made possible when the matrix $\Theta=\phi \psi$ obeys the rule of Restricted Isometric Property (RIP). For each integer $n=1,2, \ldots N$ isometry constant $\delta_{n}>$ 0 of a matrix $\phi$ can be defined as the smallest number and $|\cdot|$ is defined as the amplitude of a complex number, For signal reconstruction to be successful, $\Theta$ must satisfy [28]:

$$
\left(1-\delta_{\mathrm{n}}\right)\|\mathrm{X}\|_{2}^{2} \leq\|\Theta \mathrm{X}\|_{2}^{2} \leq\left(1+\delta_{\mathrm{n}}\right)\|\mathrm{X}\|_{2}^{2}
$$

From equation (3) we can say that a matrix $\phi$ has the RIP of orders if $\delta_{\mathrm{n}}$ is not too close to 1 . The restricted isometry constants give a measure of how much they can change the length of an Ssparse vector.

\subsection{Signal Reconstruction}

As $\alpha, \mathrm{X} \in \mathcal{P}^{\mathrm{N}}$ and $\mathrm{Y} \in \mathrm{R}^{\mathrm{M}}$ for $\mathrm{m} \ll \mathrm{n}$ there are infinitely many solutions to equation (2). If $\phi$ is incoherent to $\Psi \square$, the original CS theory proposed $\ell_{1}$ norm minimization for signal reconstruction when dealing with noise free measurements:

$$
\min \|a\|_{1} \quad \text { s. t. } \mathrm{Y}=\phi \Psi a
$$

Convex optimization techniques can be used for the sparse signal recovery with high probability. CS relied on the assumption that equation (4) provides the correct solution and is computationally not complex. However work has been done to find alternative algorithms those can be easily implemented, faster and give better reconstruction performance, such as Matching Pursuit (MP) [29] and OMP [30] algorithms, which can be fast and easily implemented. There also exist a variety of different kinds of iterative algorithms such as Compressed sampling Matching Pursuit (CoSaMP), iterative hard thresholding (IHT), and various similar thresholding algorithms for improved compressed signal reconstruction [31-37]. These algorithms are based on similar structure can be easily understood by identifying the locations of the nonzero coefficients of $\alpha$ and then estimating the values of those coefficients. 


\section{SYSTEM MODEL AND PROBLEM STATEMENT}

System model considered in our work is same as that in [38], which assumes that CR with frequency range $0-\mathrm{W}$ Hertz, as shown in Figure 1. Figures 1(a) and 1(b) corresponds to frequency frame and time frame. In time frame $0-t$ (second) is required for spectrum sensing and remaining $\mathrm{t}-\mathrm{T}$ (second) is used for transmitting data. In out work, all the received signals from different PUs and SUs are treated as one received signal, $s(t)$. Continuous signal received at the $\mathrm{RF}$ front-end of SU contains signal $\mathrm{s}_{\mathrm{i}}$ and noise $\mathrm{z}_{\mathrm{i}}$. Considered noise is a sequence of independent and identically distributed, additive zero-mean, circularly symmetric, complex Gaussian noise samples with variance $E\left\{\left|n_{i}\right|^{2}\right\}=\sigma_{n}{ }^{2}$ for all $i$. The power spectrum density (PSD) over each spectrum sub-band is independent for two neighboring subbands. In a sensing period $t-T$, the locations and the number of active subbands keep unchanged. By using sampling rate $f_{N}$ over $t$, we obtain $\alpha[n]=\alpha_{i}\left(n / f_{N}\right), n=1,2, \cdots, N$.

(a)

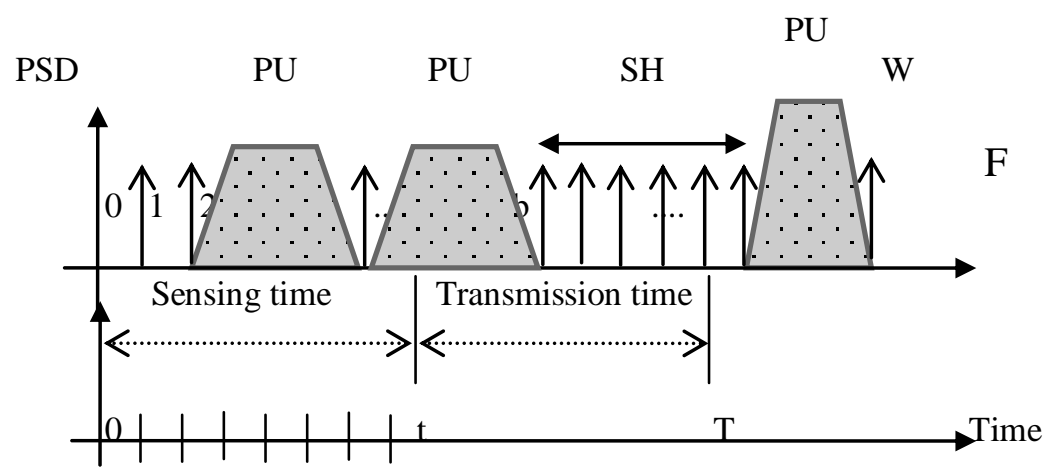

Figure 1. Wideband CR spectrum sensing model (a) Frequency Frame (b) Time Frame

By using sub-Nyquist sampling rate $\mathrm{f}_{\mathrm{S}}\left(\mathrm{f}_{\mathrm{S}}<2 \mathrm{~W}\right)$, the compressed samples $\mathrm{y}\left(\mathrm{y} \in \mathcal{P}^{\mathrm{Mx} 1}, \mathrm{M}=\tau \mathrm{f}_{\mathrm{S}}\right.$ $<<N$ ) can be written as:

$$
y=\varphi \alpha
$$

where $\varphi$ is an $\mathrm{M} \times \mathrm{N}$ sensing matrix and $\mathrm{y}$ is the measurement vector of m measurements. Based on the CS theory, efficiency of signal acquisition depends on the sparse representation of the signal. Signal $\alpha \square \epsilon \mathcal{P}^{\mathrm{N}}$ can be expressed in a sparsifying basis $\Psi=\left[\Psi_{1}, \Psi_{2}, \ldots \Psi_{\mathrm{N}}\right] \square$ as follows:

$$
\alpha=\sum x_{N} \psi_{i}
$$

where $\mathrm{x}$ is the vector of inner products, $\mathrm{x}_{\mathrm{i}}=\left\langle\alpha, \psi_{\mathrm{i}}\right\rangle$. Let $\mu(\varphi, \psi)$ be the coherence between $\varphi$ and $\psi, \mathrm{C}$ is constant, we can choose minimum number of measurements required for spectrum reconstruction:

$$
\mathrm{m} \geq \mathrm{C}_{0} \cdot \mu^{2}(\varphi, \psi) \cdot \mathrm{S} \cdot \log \mathrm{N}
$$

From (7), it can be observed that $m$ depends on the sparsifying basis. In this article, we compare the cases if the sparsifying basis is the Discrete Fourier Transform (DFT) or Discrete Cosine Transform (DCT) or Empirical Wavelet Transform (EWT). An estimate $\hat{x}$ of sparse vector can be obtained by solving the CS reconstruction problem:

$$
\hat{\mathrm{x}}=\arg \min \|\mathrm{x}\|_{1} \quad \text { s. } t . \mathrm{y}=\varphi \psi \mathrm{x}
$$

Above problem can be solved efficiently using Greedy algorithms, Tony Cai and Lie Wang at al. [39] explains OMP algorithm, which is suitable to deal with noisy signal. 
After spectrum reconstruction SUs sense the channel in order to find the spectrum holes (SHs), which can be described as the hypothesis testing problem, mathematically:

$$
\begin{array}{lll}
\mathcal{H}_{0}: & \alpha_{\mathrm{i}}=\mathrm{z}_{\mathrm{i}}, & \mathrm{i}=1,2, \ldots, \mathrm{N} \\
\mathcal{H}_{1}: & \alpha_{\mathrm{i}}=\mathrm{s}_{\mathrm{i}}+\mathrm{z}_{\mathrm{i}}, & \mathrm{i}=1,2, \ldots, \mathrm{N}
\end{array}
$$

For large values of N, it can be approximated:

$$
\alpha=\sum_{i=1}^{N}\left|\alpha_{\mathrm{i}}\right|^{2}
$$

as a Gaussian random variable by using the Central Limit Theorem, It can be verified that the mean and variance of $\alpha$ are given by, respectively:

$$
\begin{aligned}
\mathrm{E}\{\alpha\} & =\left\{\begin{array}{cc}
\sigma_{\mathrm{z}}^{2} & \text { under } \mathcal{H}_{0} \\
\sigma_{\mathrm{x}}^{2}+\sigma_{\mathrm{z}}^{2} & \text { under } \mathcal{H}_{1}
\end{array} \sigma^{\sigma_{\mathrm{z}}^{2}}\right. \\
\operatorname{Var}\{\alpha\} & =\left\{\begin{array}{cc}
\text { under } \mathcal{H}_{0} \\
\mathrm{E}\left\{|\mathrm{x}|^{4}\right\}+2 \sigma_{\mathrm{z}}^{4}-\left(\sigma_{\mathrm{x}}^{2}-\sigma_{\mathrm{z}}^{2}\right)^{2} / \mathrm{N} & \text { under } \mathcal{H}_{1}
\end{array}\right.
\end{aligned}
$$

An energy detector can be used to accumulate the energy $E_{=} \sum_{i \in \text { sub-band } b} \hat{x}(i)$ received in subband $b$. The SU decides the spectrum availability by:

$$
\text { (b) }= \begin{cases}\mathcal{H}_{0} & \alpha_{\mathrm{i}} \geq \lambda \\ \mathcal{H}_{1} & \alpha_{\mathrm{i}}<\lambda\end{cases}
$$

The threshold $\lambda$ is a design parameter for the CR receiver system. The choice of $\lambda$ provides a trade-off between maximizing SU throughput and minimizing interference to the PU. Two probabilities of interest are defined as follows

$$
\begin{array}{ll}
\text { Probability of false alarm: } & \mathrm{P}_{\mathrm{f}}=\mathrm{P}_{\mathrm{r}}\left\{\alpha \geq \lambda \mid \mathcal{H}_{0}\right\} \\
\text { Probability of detection: } & \mathrm{P}_{\mathrm{d}}=\mathrm{P}_{\mathrm{r}}\left\{\alpha<\lambda \mid \mathcal{H}_{1}\right\}
\end{array}
$$

After spectrum sensing, CR adopts NC-OFDM technique that decomposes wideband into orthogonal sub-channels. In NC-OFDM based CR systems, as shown in Figure 2, the subchannels are activated when the spectrum is idle and when it is not available corresponding subchannels are deactivated (set to zero). The data stream is only assigned to active sub-carriers and pilot design is performed accordingly.

In wide-band wireless communication systems, the actual bandwidth of the system is usually larger than the coherence bandwidth and the channel is usually frequency selective fading channel. The vector is S-sparse, Discrete Fourier Transform (DFT) size is N, active sub-carriers are $\mathrm{Q}$ and pilot sub carriers $\left(\mathrm{c}_{\mathrm{p}}\right)$ are $\mathrm{P}(\mathrm{P} \leq \mathrm{Q})$. The cyclic prefix $(\mathrm{CP})$ length is greater than the maximum possible path delay. OFDM symbol data $X(n)$ contains mapping signals and pilot signals. After removing CP, DFT is applied to the received signal $\mathrm{y}_{\mathrm{n}}$ for $\mathrm{n} \epsilon[0, \mathrm{~N}-1]$ to obtain $\mathrm{k} \epsilon$ $[0, \mathrm{~N}-1]$. The received signal is $\mathrm{N} \times 1$ sample vector. The discrete-time channel model is:

$$
\mathrm{h}(\mathrm{n})=\sum_{\mathrm{l}=0}^{\mathrm{l}-1} \mathbf{h}_{\mathbf{l}} \boldsymbol{\delta}(\mathbf{n}-\mathbf{l})
$$

where the impulse response vector of the channel $h=\left[h_{0}, h_{1}, \ldots, h_{1-1}\right]^{\mathrm{T}}$ remains unchanged in multiple OFDM symbol period of time reflects the slow time variation of the channel. 


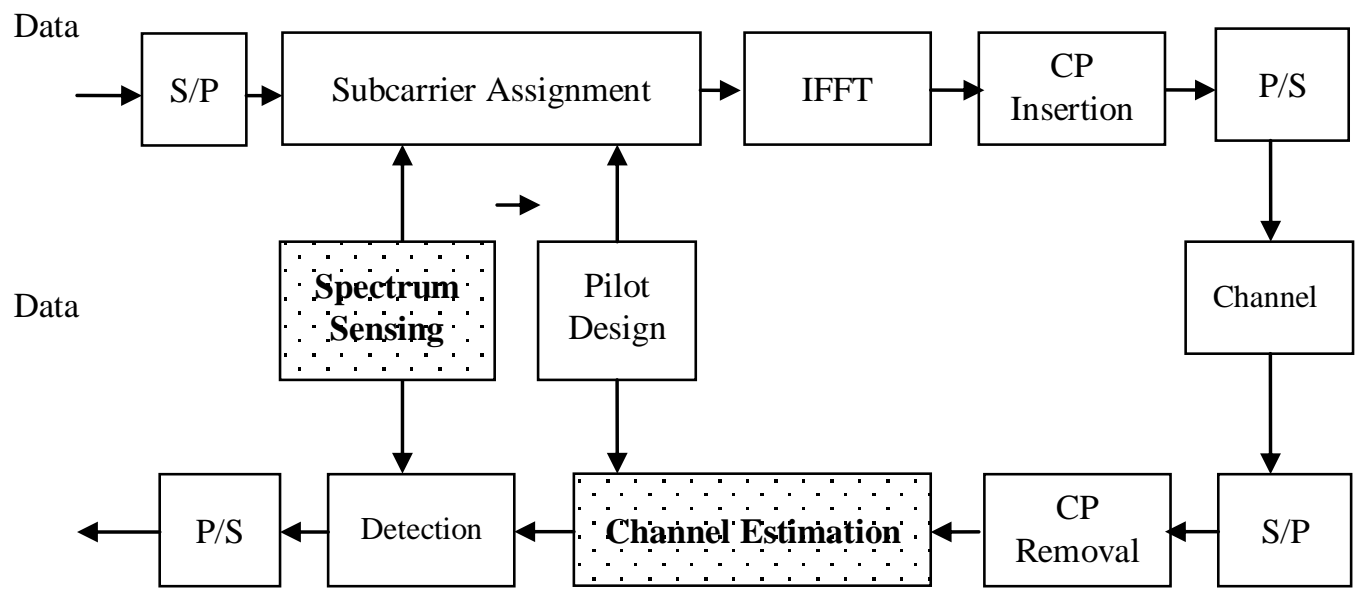

Figure 2. NC-OFDM based CR system

where the impulse response vector of the channel $h=\left[h_{0}, h_{1}, \ldots, h_{1-1}\right]^{T}$ remains unchanged in multiple OFDM symbol period of time reflects the slow time variation of the channel. The relation between the transmitted pilots and received pilots can be written as:

$$
\left.\left.\left.\begin{array}{c}
\mathrm{y}\left(\oint_{\mathrm{p}_{1}}\right) \\
\mathrm{y}\left(\mathrm{c}_{\mathrm{p}_{2}}\right) \\
\vdots \\
\mathrm{y}\left(\mathrm{p}_{\mathrm{pk}}\right)
\end{array}\right]=\left[\begin{array}{cccc}
\mathrm{x}\left(\mathrm{c}_{\mathrm{p}_{1}}\right) & 0 & \ldots & 0 \\
0 & \ddots & & 0 \\
\vdots & & \ddots & \vdots \\
0 & 0 & \ldots & \mathrm{x}\left(\mathrm{c}_{\mathrm{p}_{\mathrm{k}}}\right)
\end{array}\right] \cdot \mathrm{F}_{\mathrm{K} \times \mathrm{L}} \cdot \begin{array}{r}
\mathrm{h}(1) \\
\mathrm{h}(2) \\
\mathrm{h}(\mathrm{L})
\end{array}\right] \begin{array}{r}
\mathrm{z}(\mathfrak{q}) \\
\mathrm{z}(2) \\
\vdots \\
\mathrm{z}(\mathrm{k})
\end{array}\right](18)
$$

where $\mathrm{Z}$ is additive White Gaussian Noise (AWGN) and is $\mathrm{F}_{\mathrm{K} \times \mathrm{L}}$ is a DFT sub matrix given by:

$$
\mathrm{F}_{\mathrm{K} \times \mathrm{L}}=\frac{1}{\sqrt{N}}\left[\begin{array}{cccc}
1 & \mathrm{w}^{\mathrm{c}_{\mathrm{p}_{1}}} & \ldots & \mathrm{w}^{\mathrm{c}_{\mathrm{p}_{1}} \cdot(\mathrm{L}-1)} \\
1 & \ddots & & \mathrm{w}^{\mathrm{c}_{\mathrm{p}_{2}} \cdot(\mathrm{L}-1)} \\
\vdots & & \ddots & \vdots \\
1 & \mathrm{w}^{\mathrm{c}_{\mathrm{K}}} & \ldots & \mathrm{w}^{\mathrm{c}_{\mathrm{p}_{\mathrm{K}}}(\mathrm{L}-1)}
\end{array}\right]
$$

where $\mathrm{w}^{\mathrm{nl}}=\mathrm{e}^{-\mathrm{j} \frac{2 \pi \mathrm{nl}}{\mathrm{N}}}$. Let $\mathrm{A}=\mathrm{XF}_{\mathrm{K} \times \mathrm{L}}$. Then (18) can be written as:

$$
\mathrm{y}=\mathrm{Ah}+\mathrm{Z}
$$

$\mathrm{R} \times \mathrm{N}$ matrix $\mathrm{S}$ selects the location of $\mathrm{P}$ pilot from the $\mathrm{N}$ subcarriers. $\mathrm{N} \times \mathrm{N}$ matrix $\mathrm{S}$ selects $\mathrm{R}$ rows corresponds to the pilot position from the unit matrix. The pilot signal received is:

$$
\mathrm{y}_{\mathrm{p}}=\mathrm{X}_{\mathrm{p}} \mathrm{H}_{\mathrm{p}}+\mathrm{Z}_{\mathrm{p}}=\mathrm{X}_{\mathrm{p}} \mathrm{W}_{\mathrm{p}} \mathrm{h}+\mathrm{Z}_{\mathrm{p}}
$$

where $\mathrm{R} \times 1$ matrix $\mathrm{y}_{\mathrm{p}}=\mathrm{Sy}, \mathrm{R} \times \mathrm{R}$ matrix $\mathrm{X}_{\mathrm{p}}=\mathrm{SXS}, \mathrm{R} \times \mathrm{L}$ matrix $\mathrm{W}_{\mathrm{p}}=\mathrm{SW}, \mathrm{R} \times 1$ matrix $\mathrm{n}_{\mathrm{P}}=$ S. In (21) $\mathrm{y}_{\mathrm{p}}, \mathrm{X}_{\mathrm{p}}$ and $\mathrm{W}_{\mathrm{p}}$ are known. We can re-obtain system channel relation obtained in (20) by:

$$
\mathrm{H}_{\mathrm{lk}}=\sum_{m=0}^{K-1} \sum_{i=0}^{L=1} F[m, i] e^{-j 2 \Pi\left(\frac{k m}{K}-\frac{l_{i}}{i}\right)}
$$


where

$$
\mathrm{F}[\mathrm{m}, \mathrm{i}]=\mathrm{X}_{\mathrm{h}}(\mathrm{m}, \mathrm{i}) \cdot A_{\Upsilon, g}^{*}\left(\mathrm{~m}, \mathrm{i} / \mathrm{N}_{\mathrm{r}}\right)
$$

with the cross ambiguity function [40] $\mathrm{A}_{\gamma, g}(\mathrm{~m}, \varepsilon)=\sum_{n=\infty}^{-\infty} \gamma[n] g[n-m] e^{-2 \Pi \varepsilon n}$. The purpose of channel estimation is to find the channel frequency response $\mathrm{H}$. Since the channel delay spread is much larger than sampling period, particularly for OFDM systems with over sampling, most components of $h$ are either zero or very close to zero, which implies that $h$ is sparse. With this a priori condition, CS algorithm can be applied to estimate $h$.

\section{COMPRESSED SPECTRUM SENSING}

Consistent with the theory of CS discussed in Section 4, an N point signal $\mathrm{x}$, or its $\mathrm{S}$ sparse representations can be reconstructed from only m measurements. According to equation (1) and (2):

$$
\mathrm{y}=\varphi \alpha=\varphi \psi_{\mathrm{s}} \mathrm{x}=\varphi \psi_{\mathrm{s}} \mathrm{F}^{-1} \mathrm{X}
$$

where $\mathrm{F}^{-1} \mathrm{X}$ is the inverse Fourier transform of $\mathrm{x}$. From (7), the minimum number of measurements depends on the sparsifying basis $\psi_{\mathrm{s}}$. We apply method, empirical wavelet transform scheme to find sparse representations of the spectrum in the context of compressive sensing. The discrete signal $\mathrm{x}$ is not known prior to sensing so first we calculate an estimated version of the signal $\hat{\mathrm{x}}$ using an appropriate conventional sparsifying matrix. Next, we apply empirical wavelet scheme. Jerome Gilles [43] proposed a method is to build a family of wavelets adapted to the processed signal. The process is equivalent to building a set of band pass filters. Filters' support depends on where information in the spectrum of analyzed signal is located. Consider a spectrum $[0, \pi]$ as shown in Figure 2, which is segmented into I segments; $\omega_{\mathrm{i}}$ is the limit between each segment $\Lambda_{\mathrm{i}}$. $\eta_{\mathrm{i}}$ is the transition phase of width $2 \tau_{\mathrm{i}}$.

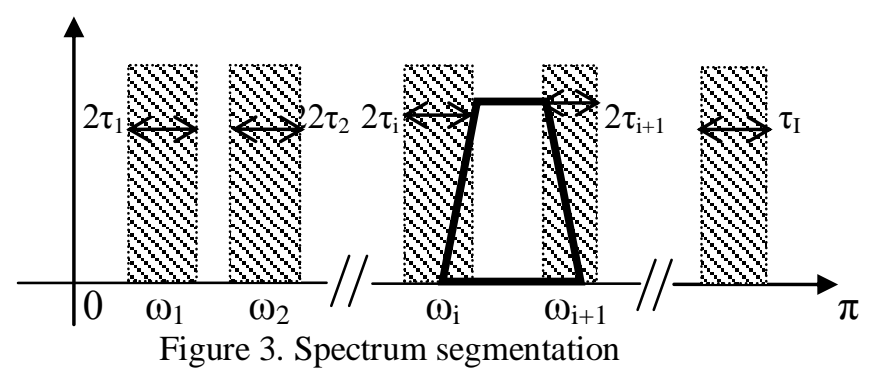

The empirical wavelets are defined as band pass filters on each segment. Empirical scaling function and empirical wavelets are defined by expressions, (25) and (26) respectively

$$
\Phi_{\mathrm{i}}(\omega)=\left\{\begin{array}{cc}
1 & \text { if }|\omega| \leq \omega_{\mathrm{i}}-\tau_{\mathrm{i}} \\
\cos \left[\pi \beta / 2\left(1 / 2 \tau_{i}\left(|\omega|-\omega_{\mathrm{i}}+\tau_{\mathrm{i}}\right)\right)\right] \\
\text { if } \omega_{\mathrm{i}}-\tau_{\mathrm{i}} \leq|\omega| \leq \omega_{\mathrm{i}}+\tau_{\mathrm{i}} \\
0 \quad \text { otherwise }
\end{array}\right.
$$




$$
\Psi_{\mathrm{i}}(\omega)=\left\{\begin{array}{c}
1 \quad \text { if } \omega_{i}+\tau_{i} \leq|\omega| \leq \omega_{\mathrm{i}+1}-\tau_{i+1} \\
\cos \left[\beta \pi / 2\left(\frac{1}{\tau_{i+1}}\left(|\omega|-\omega_{i+1}+\tau_{i+1}\right)\right)\right] \\
\text { if } f \omega_{i}+\tau_{i} \leq|\omega| \leq \omega_{i+1}-\tau_{i+1} \\
\cos \left[\beta \pi / 2\left(\frac{1}{\tau_{i+1}}\left(|\omega|-\omega_{i+1}+\tau_{i+1}\right)\right)\right] \\
\text { iff } \omega_{i}+\tau_{i} \leq|\omega| \leq \omega_{i+1}-\tau_{i+1} \\
0
\end{array}\right.
$$

The function $\beta_{x}$ is an arbitrary $C^{\mathrm{k}}([0,1])$ function [44] such that

$$
\beta(x)= \begin{cases}0 & \text { if } x \leq 0 \\ \text { and } \beta(x)+\beta(x+1) & =1 \forall x \in[0,1] \\ 1 & \text { if } x \geq 1\end{cases}
$$

Using equation (24) problem reconstruction of $\hat{\mathrm{x}}$ can be converted into the problem of reconstruction of $\widehat{\mathrm{X}}$ :

$$
\hat{x}=\arg \min \|\widehat{\mathrm{X}}\|_{0} \quad \text { s.t. } \mathrm{y}=\left(\varphi \mathrm{F}^{-1}\right) \widehat{\mathrm{X}}
$$

It can be seen that (28) is a non-convex problem as there can be many optimal solutions. Equation (28) has a unique solution when:

$$
\widehat{x}=\arg \min \|\widehat{\mathrm{X}}\|_{1} \quad \text { s.t. } \mathrm{y}=\left(\varphi \mathrm{F}^{-1}\right) \widehat{\mathrm{X}}
$$

To deal with the signals with noise components, some variants of LASSO algorithm can be developed by minimizing the usual sum of squared errors [41, 42]:

$$
\hat{x}=\arg \min \|\widehat{\mathrm{X}}\|_{0} \quad \text { s.t. }\left\|\varphi \mathrm{F}^{-1} \widehat{\mathrm{X}}-\mathrm{y}\right\|_{2} \leq \varepsilon
$$

where $\varepsilon$ is recovery error threshold. The EWT-CSS problem can be solved with a two-step scheme: First, use compressed measurements y to estimate the sparse sequence and second, reconstruct signal $\hat{\mathrm{x}}$ according to $\psi_{\mathrm{s}}$.

$\ell_{2}$ norm approach is used to terminate the signal acquisition automatically at CR receiver. As discussed in Section 3 time frame is divided into sensing interval $(0-t)$ and transmitting interval $(\mathrm{t}-\mathrm{T})$, if we decrease the sensing time, we can increase the time interval to transmit data. First, we divide complete sensing time interval $t$ into I mini time slots. $R_{i}$ is training subset, $T_{i}$ is testing subset and $\mathrm{V}_{\mathrm{i}}$ is the verification parameter, for best spectrum recovery:

$$
\mathrm{V}_{\mathrm{i}}=\left\|\overrightarrow{T_{l}}-\psi_{\mathrm{i}} \mathrm{F}^{-1} \widehat{\mathrm{X}}_{\mathrm{i}}\right\|_{2}^{2}
$$

Using equation (2) and (10), $\mathrm{R}_{\mathrm{i}}$ and $\mathrm{T}_{\mathrm{i}}$ can be written as correspondingly

$$
\begin{aligned}
& \mathrm{R}_{\mathrm{i}}=\varphi_{\mathrm{i}} \mathrm{x}_{\mathrm{i}}+\mathrm{n}=\varphi_{\mathrm{i}} \mathrm{F}^{-1} \overrightarrow{\mathrm{X}}_{\mathrm{i}}+\mathrm{n}_{\mathrm{i}} \\
& \mathrm{T}_{\mathrm{i}}=\psi_{\mathrm{i}} \mathrm{x}_{\mathrm{i}}+\mathrm{n}=\psi_{\mathrm{i}} \mathrm{F}^{-1} \overrightarrow{\mathrm{X}}_{\mathrm{i}}+\mathrm{n}_{\mathrm{i}}
\end{aligned}
$$

where $\varphi_{i}$ is a $r_{i} \times N$ measurement matrix, $\psi_{i}$ is a $t_{i} \times N$ testing matrix, and $n$ denotes the noise in measurements. 


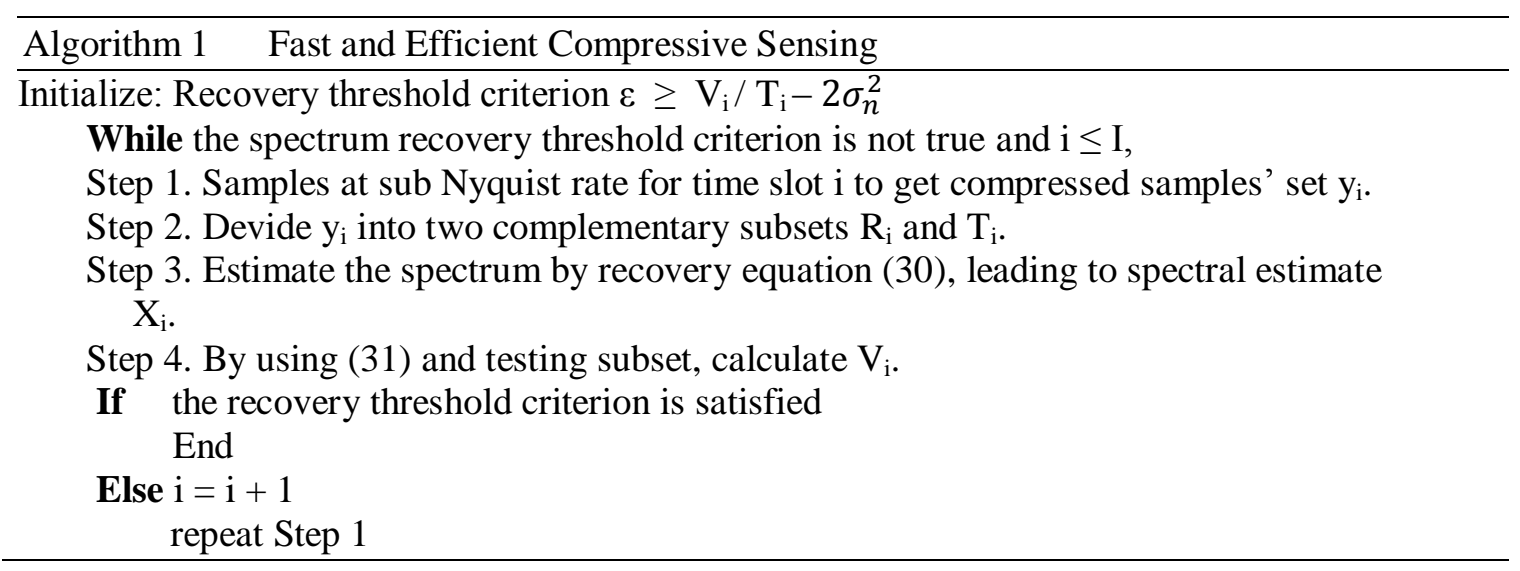

Repeating this procedure, a sequence of spectrum estimates, i.e., $\widehat{X}_{1}, \widehat{X}_{2}, \ldots, \widehat{X}_{1}$ will be obtained, and by increasing measurements $\mathrm{m}_{\mathrm{i}}$, we can get a a better estimate of the spectrum with minimum spectrum recovery error ||$X_{i}-\widehat{X}_{i} \mid$. Terminating the signal acquisition automatically and by using the remaining slots for transmission, leads to fast and efficient spectrum sensing and improved system throughput.

\section{SPARSE CHANNEL ESTIMATION}

In the CS model discussed in Section 3, the signal $\mathrm{x}$ is not directly measured, but projected onto the observation matrix $\xi=\left[\xi_{1}, \xi_{2}, \ldots \xi_{\mathrm{M}}\right]$, and then the sampling vector y is obtained. The matrix form is

$$
\mathrm{y}=\xi \mathrm{x}
$$

where $\mathrm{x}$ is an $\mathrm{N} \times 1$ vector, $\xi$ is an $\mathrm{M} \times \mathrm{N}$ matrix, and $\mathrm{y}$ is an $\mathrm{M} \times 1$ sampling vector. If signal $\mathrm{x}$ is sparse in the transform basis $\psi^{\mathrm{T}}=\left[\psi_{1}, \psi_{2}, . . \psi_{\mathrm{N}}\right]$, it can be expressed as follows:

$$
\mathrm{x}=\sum_{\mathrm{k}=1}^{\mathrm{N}} \psi_{\mathrm{k}} \alpha_{\mathrm{k}}=\psi \alpha
$$

where $\alpha$ is an $\mathrm{N} \times 1$ vector, $\psi$ is an $\mathrm{N} \times \mathrm{N}$ sparse vector. (8) is substituted into (7):

$$
\mathrm{y}=\xi \mathrm{x}=\xi \psi \alpha=\Theta \alpha
$$

where $\mathrm{M} \times \mathrm{N}$ matrix $\xi=\psi \Theta$. The observation dimension $\mathrm{M}$ is far less than the signal dimension $\mathrm{N}$, so the signal $\mathrm{x}$ can't be solved with $\mathrm{M}$ sampling values of $\mathrm{y}$. As $\alpha$ is sparse, with the sparse decomposition algorithm based on the theory of sparse decomposition, $\alpha$ can be solved by the inverse problem of solving (36), and be substituted into (35) to obtain the signal x. Consider the baseband channel model of (4).

Due to the channel fading and noise, to determine the length of training sequence $\Theta$ is important in terms of both spectrum efficiency and estimation robustness. Therefore, the length $\mathrm{N}$ of $\Theta$ must satisfy

$$
\mathrm{N} \geq \mathrm{C} \cdot \mathrm{S} \cdot(\log \mathrm{L})^{4} \mu_{\mathrm{x}}^{2}
$$

where $C$ is a constant and $\mu_{x}=\sqrt{L} \max _{i, j}\left|X_{i, j}\right|$ which is known as the maximum coherence between the $i_{\text {th }}$ column and $j_{\text {th }}$ column of $\Theta$. 
OMP algorithms suggests the reconstruction under the conditions of a given iteration number, as the iterative process is forced to stop, OMP algorithm needs a lot of linear measurement to ensure accurate reconstruction. The basic idea of the OMP algorithm is to select the columns of $\Theta$ with greedy iterative algorithm, make sure the correlative value between the columns selected in each iteration and the current redundant vector is maximum, and then subtract the correlative value from the sampling vector and repeat iteration until the number of iterations achieves the sparse degree $S$. Here, $\Theta$ is sensing matrix, y is sampling vector, $\mathrm{S}$ is sparse degree, $\mathrm{r}$ is residual and $\Lambda$ is the index set . Output of the OMP algorithm is the S-sparse approximation $\widehat{\alpha}$ of $\alpha$.

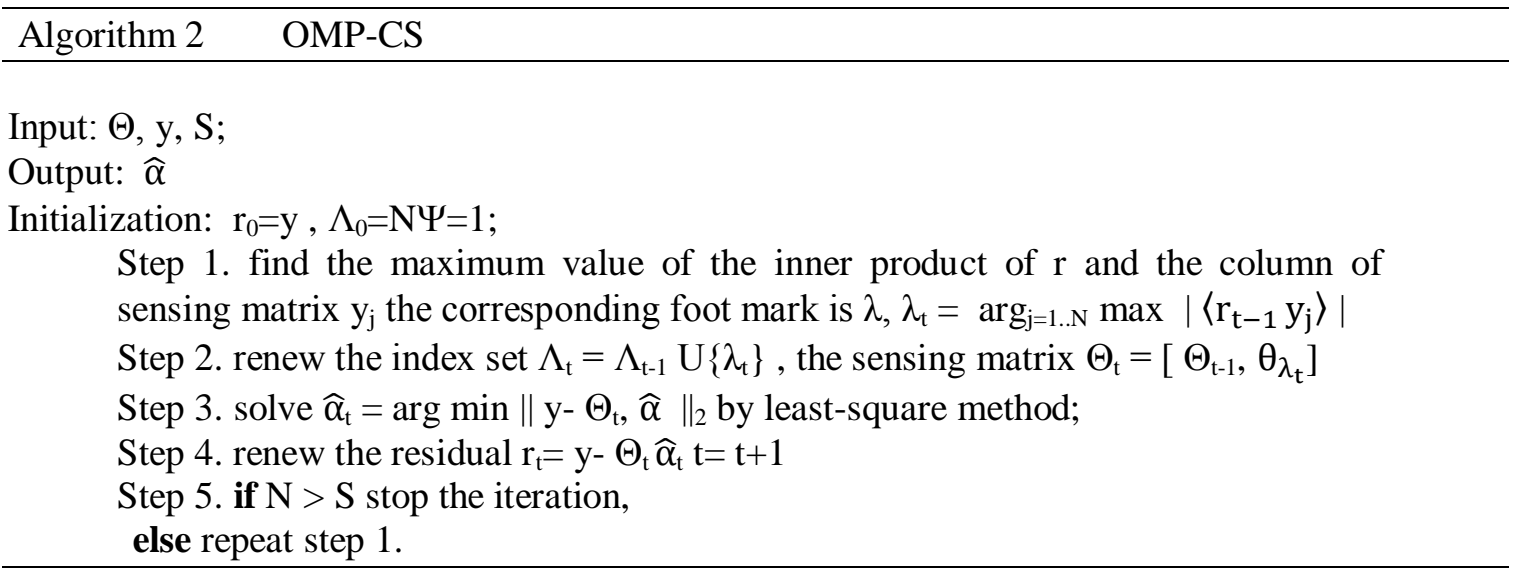

OMP algorithm selects an atom in each iteration to update the atom collection, which will certainly pay a large time for reconstruction. The number of iterations is closely related to sparse degree $\mathrm{S}$ and the number of samples $\mathrm{M}$, with their increase, time consumption will also increase significantly.

Problem with Algorithm 2 is that it is not adaptive, pre-estimate of the sparse degree of the sparse signal is needed and the reconstruction accuracy is not satisfactory. In reality, the sparse degree of the sparse channel is usually unknown. Nam Naguyen et al. [30] proposed extended OMP-CS algorithm in order to improve the accuracy of reconstruction, and make the algorithm adaptive. In the ExtOMP-CS algorithm, one key issue is how to choose the step size. Unlike the OMP-CS algorithm, the iteration times of ExtOMP-CS algorithm is not certain and is related to step size and computational complexity and computational time are higher in the ExtOMP-CS algorithm than OMP-CS algorithm.

An extension to OMP algorithms is the Compressed Sampling Matching Pursuit (CoSaMP) algorithm [45]. The basis of the algorithm is OMP but CoSaMP can be shown to have tighter bounds on its convergence and performance.

\section{Algorithm 3 Compressed Sampling Matching Pursuit Algorithm}

Input: $\mathrm{S}, \mathrm{y}, \phi$

1. $\mathrm{x}(0) \leftarrow 0$

2. $\mathrm{v} \leftarrow \mathrm{y}$

3. $\mathrm{k} \leftarrow 0$

4. while Halting condition false do

5. $\mathrm{k} \leftarrow \mathrm{k}+1$

6. $\mathrm{z} \leftarrow \phi^{\mathrm{T}} \mathrm{v} \quad$ : signal proxy

7. $\Omega \leftarrow \mathrm{z}^{2 \mathrm{~S}}$ : find the largest $2 \mathrm{~S}$ components of the signal proxy (Identification) 
8. $\Gamma \leftarrow \Omega$ supp $\left(\mathrm{x}^{(\mathrm{k}-\mathrm{l})}\right) \quad$ : merge the support of the signal proxy with the support of the solution from the previous iteration (Support Merge)

9. $\bar{x} \leftarrow \arg \min \|(\phi x-y)\|_{2}:$ estimate a solution via least squares with the constraint that the solution lies on a particular support (Estimation)

10. $\mathrm{x}^{\mathrm{k}} \leftarrow \mathrm{x}^{\mathrm{s}} \quad$ : takes the solution estimate and compresses it to the required support (Pruning)

11. $\mathrm{v} \leftarrow \mathrm{y}-\phi \mathrm{x} \quad:$ update the sample, namely the residual in F-space (Sample Update)

12. end

13. while $\hat{x} \leftarrow \mathrm{x}(\mathrm{k})$

Output: $\hat{\mathrm{x}} \quad$ : such that it is S-sparse and $\mathrm{y}=\phi \mathrm{x}$

If N-length training sequence $X$ satisfies the RIP and $\delta_{2 S} \leq \sqrt{2}-1$, for any $2 S$-sparse channel vector $\mathrm{h}$, it produces the channel estimator $\hat{\mathrm{h}}$ that satisfies

$$
\|\mathrm{h}-\hat{\mathrm{h}}\|_{2} \leq \mathrm{C} \max \left\{\varepsilon, 1 / \sqrt{\mathrm{S}}\left\|\mathrm{h}-\hat{\mathrm{h}}_{2 \mathrm{~S}}\right\|_{1}+\|\mathrm{z}\|_{2}\right\}
$$

\section{Performance AnAlysis AND Simulation ReSUlt}

In NC-OFDM the power spectral density (PSD) of $\mathrm{P}^{\text {th }}$ subcarrier signal is characterized of the form

$$
\Gamma_{\mathrm{k}}(\mathrm{f})=\mathrm{K} \cdot \operatorname{sinc}^{2}\left(\left(\mathrm{f}-\mathrm{f}_{\mathrm{P}}\right) \mathrm{T}_{\mathrm{S}}\right)
$$

where $\mathrm{K}$ is the signal level, $\mathrm{f}_{\mathrm{k}}$ is the sub-carrier center frequency, and $\mathrm{T}_{\mathrm{S}}$ is the OFDM symbol duration and $\mathrm{T}_{\mathrm{G}}$ is guard interval. Assuming independent symbols in different subcarriers, the PSD of an NC-OFDM signal is obtained as

$$
\Gamma(\mathrm{f})=\sum_{\mathrm{P}} \Gamma_{\mathrm{P}}(\mathrm{f})
$$

where index $\mathrm{P}$ is the number of active subcarriers. In this paper we are considering a spectrum (0$500 \mathrm{MHz}$ ) with four sub-bands located at $30 \mathrm{MHz}-70 \mathrm{MHz}, 120 \mathrm{MHz}-180 \mathrm{MHz}, 300 \mathrm{MHz}-$ $340 \mathrm{MHz}, 420 \mathrm{MHz}-460 \mathrm{MHz}$ and their corresponding power spectral density (PSD) levels are in the range of $0.0277-0.1126,0.0157-0.0988,0.0588-0.1294$, and $0.0381-0.1201$, as shown in Fig. 3. The sub-band $b$ is in the frequency range $\left[f_{b}-B_{b} / 2, f_{b}+B_{b} / 2\right]$, where $B_{b}=10-30 \mathrm{MHz}$ and $\mathrm{f}_{\mathrm{b}}$ is in $\left[\mathrm{B}_{b} / 2, \mathrm{~W}-\mathrm{B}_{\mathrm{b}} / 2\right]$. Each time frame has length $\mathrm{T}=10 \mu \mathrm{s}$ and $\mathrm{t}$ is $5 \mu \mathrm{s}$, which is divided into $I=20$ mini time slots. Instead of using the Nyquist sampling rate $f_{N}=1 \mathrm{GHz}$, we are sampling at the sub-Nyquist rate $\mathrm{f}_{\mathrm{S}}=250 \mathrm{MHz}$. The number of compressed samples in conventional CS system is $M=f_{s} t=1250$, whereas $N=f_{N} t=10,000$. Calculating $\widehat{x}_{1}$ and $T_{i}$ after each time slot we calculate the verification parameter using (31). $V_{i}$ becomes very close to $2 \sigma^{2}$ at time slot $\mathrm{L}=9$, so using the presented algorithm we can recover the spectrum at lesser time slots. All the simulations are carried out in the MATLAB 2014.

\subsection{RECOVERED PSD}

As can be observed from Figure 3, the wideband signal contains the PSD. Using algorithm 1 for fast and efficient compressed sensing, wideband spectrum can be successfully recovered, as shown in Figure 4 and terminate the signal acquisition at comparatively lesser time slots leading to a fast efficient spectrum sensing. 
International Journal of Computer Networks \& Communications (IJCNC) Vol.7, No.5, September 2015

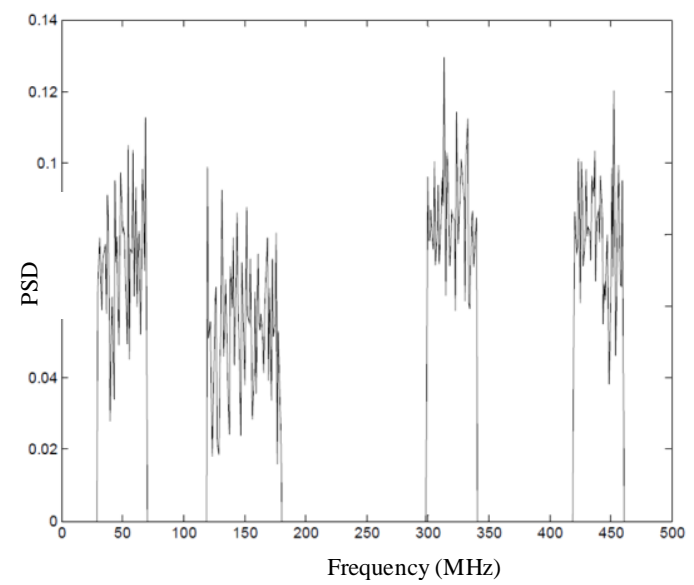

Figure 4. Wideband Spectrum $\overrightarrow{\mathrm{X}}$

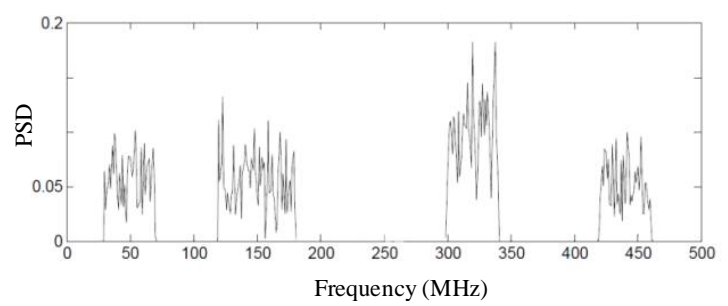

Figure 5. Reconstructed Spectrum $\widehat{X}$

\subsection{PROBABILITY OF DETECTION PERFORMANCE}

The energy detection scheme using (15) and (16) is used to detect spectrum hole by using the reconstructed spectrum. The performance of different spectrum-sensing algorithms based on DF, EWT and DCT, is compared by MATLAB simulation via $P_{d}$ for constant $P_{f}=0.05$.

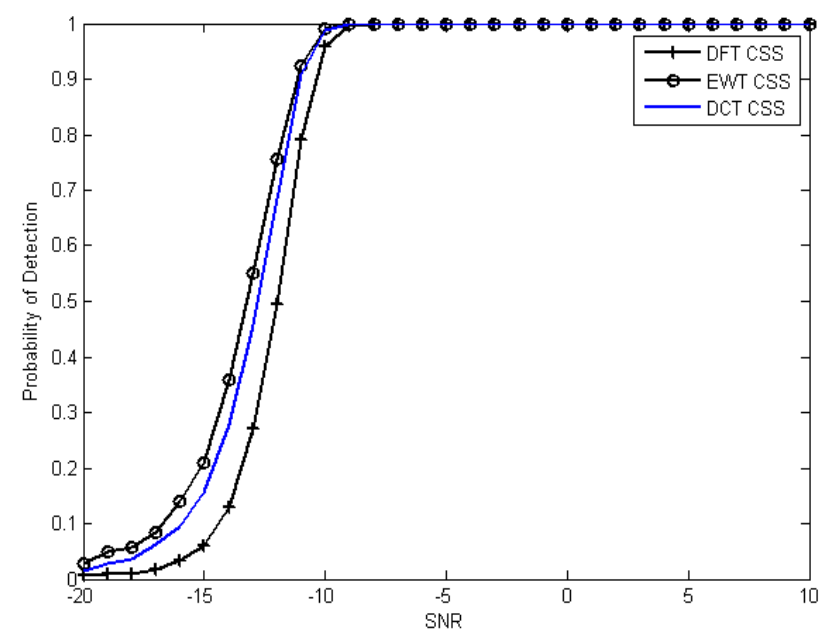

Figure 6. Performance comparison of DFT CSS, DCT CSS and proposed EWT CSS spectrum-sensing algorithms 


\subsection{ESTIMATION ERROR}

The MSE performance of the proposed estimation method will be evaluated by simulations and compared with the MSE performance of LS. It is obvious that smaller MSE means more accurate channel estimation and vice versa. MSE is defined as

$$
\mathrm{MSE}=1 / \mathrm{M} \sum_{\mathrm{m}=1}^{\mathrm{M}}\left\|\mathrm{h}-\hat{\mathrm{h}}_{\mathrm{m}}\right\|_{2}^{2}
$$

Figure 7 depicts the MSE versus SNR. It can be seen from the figure, that the CS-based scheme significantly outperforms the least-squares method. Compared with the least square scheme with 1024 pilots, CS based scheme with 511 pilots shows only a small performance degradation.

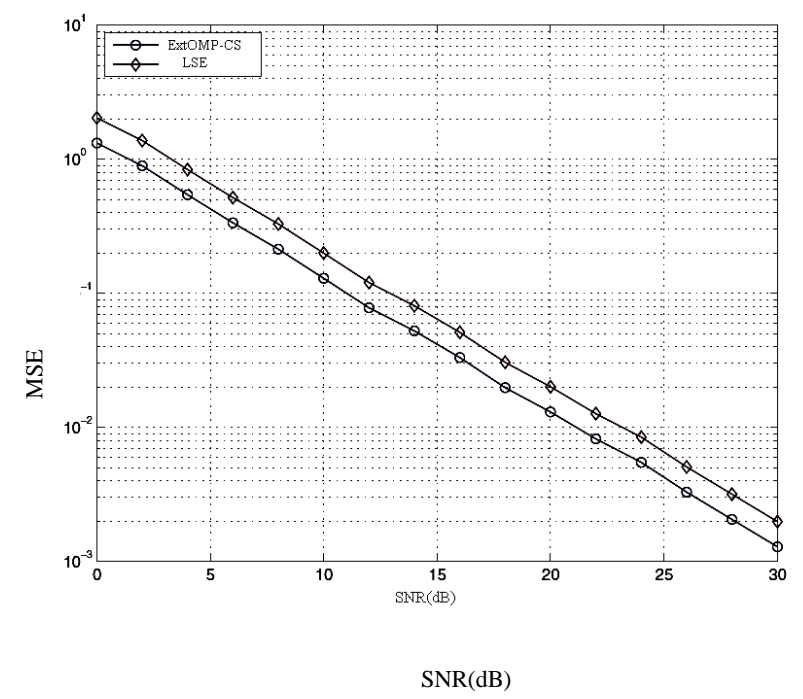

Figure 7. Performance of the channel estimate MSE for different SNRs

\subsection{BER PERFORMANCE}

We are considering an OFDM-based CR system with $\mathrm{M}=1024$ subcarriers, after spectrum sensing without any false alarm or missing detection and deactivating those subcarriers occupied by PUs, we assume that there are 512 remaining OFDM subcarriers for SUs, including three noncontiguous subcarrier blocks, i.e., $\{1,2, \ldots, 256\},\{513,514, \ldots, 640\}$ and $\{897,898, \ldots$, $1024\}$, with the number of subcarriers in each block being 256,128 , and 128 , respectively. A sparse multipath channel $\mathrm{h}$ is considered with $\mathrm{L}=60$ taps where 5 non-zero taps are placed randomly. We now evaluate the channel estimation performance using the designed pilot patterns.

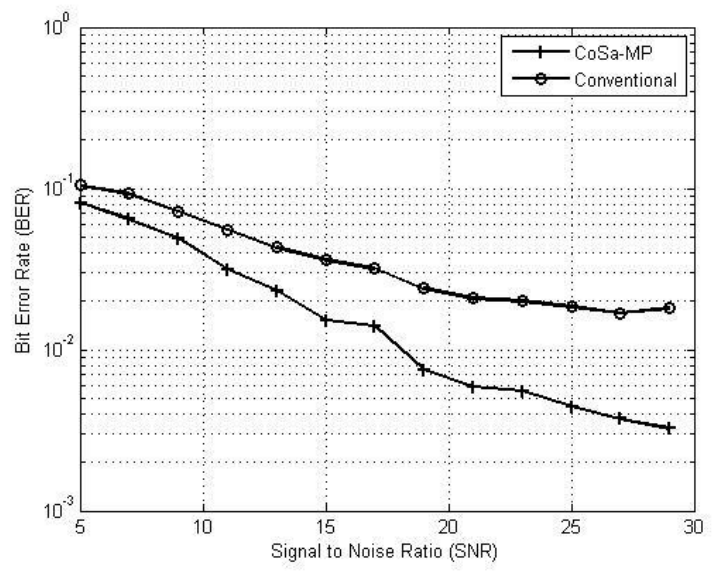

Figure 8. Comparison of BER Performances 
Figure 8 shows BER performance of two schemes- proposed CS channel estimation and channel estimation scheme based on LS. Improved BER performance of the proposed CS based scheme can be seen from the above figure, over the conventional LS based scheme.

\section{CONCLUSION}

CS is a very promising technique in wireless communication networks. However, the studies on the applications of CS are just in fewer areas. Even in these areas, a lot of problems are still not been fully settled, limiting the performance of CS. In CR network systems, if the number of channels, is not large enough, the requirement of sparsity cannot be guaranteed, which limits the advantages of CS. In this article, we demonstrated the application of CS in CR networks and based on the advantages of the proposed scheme, the problem of designing a high-performance $\mathrm{CR}$ receiver indicates that the approach should work both for spectrum sensing and channel estimation. On future work, we will consider an adaptive CS algorithm which senses the sparseness of the signal, random noise and other unexpected interferences and can differentiate between primary user and secondary user signals.

\section{REFERENCES}

[1] V. Kotelnikov, "On the carrying capacity of the "ether' and wire in telecommunications," Material for the First All-Union Conference on Questions of Communication, Izd. Red. Upr. Svyazi RKKA, Moscow, Jan. 1933.

[2] H. Nyquist, "Certain Topics in Telegraph Transmission Theory," Proceedings of IEEE, vol. 90, no.2, pp. 280-305, Fab. 2002.

[3] C. E. Shannon, "Communication in the presence of noise," Proceedings of IEEE, vol. 86, no.2, pp. 447-457, Fab. 1998.

[4] D. Donoho, "Compressed sensing," IEEE Transaction on Information Theory, vol. 52, no. 4, pp. 4036-4048, 2006.

[5] E. Cand es and T. Tao, "Near optimal signal recovery from random projections: Universal encoding strategies?” IEEE Transaction on Information Theory, vol. 52, no. 12, pp. 5406-5425, 2006

[6] E. Cand es, J. Romberg, and T. Tao, "Robust uncertainty principles: Exact signal reconstruction from highly incomplete frequency information," IEEE Transaction on Information Theory, vol. 52, no. 2, pp. 489-509, 2006.

[7] P. Indyk, M. Ruzic, "CS: Compressed Genotyping, DNA Sudoku - Harnessing high throughput sequencing for multiplexed specimen analysis Near-Optimal Sparse Recovery in the L1 Norm," Foundations of Computer Science, pp. 199-207, Oct. 2008

[8] M. Davenport, "The Fundamentals of Compressive Sensing," SigView, April 12, 2013.

[9] Y. L. Polo, Y. Wang, A. Pandharipande and G. Leus, "Compressive Wide-Band Spectrum Sensing," International Conference on Acoustics, Speech, and Signal Processing, 2009.

[10] Z. Tian and G. B. Giannakis, "Compressed sensing for wideband cognitive radios," Proceedings of IEEE ICASSP, 2007.

[11] C. R. Berger, S. Zhou, J. C. Preisig, and P. Willett, "Sparse channel estimation for multicarrier underwater acoustic communication: From subspace methods to compressed sensing," Proc. of MTS/IEEE OCEANS conference, 2013.

[12] X Jiang, WJ Jeng, E Cheng, "A fast algorithm for sparse channel estimation via orthogonal matching pursuit," IEEE 73rd Vehicular Technology Conference (VTC Spring), Budapest, pp 1-5, 2011.

[13] Mandal, M. K., Zhi Ning Chen, "Compact dual band and ultra-wideband loop antennas," IEEE Transactions on Antennas and Propagation, vol. 59, no. 8, pp. 2774-2779, Aug. 2011.

[14] S. Khalid, Wong Pen Wen, Lee Yen Cheong, "Design of highly selective ultra wideband bandpass filters using multiple resonance resonator," IEEE International RF and Microwave Confrence, pp.161-164, 2013.

[15] Zhi Tian and G. B. Giannakis, "Compressed sensing for wideband cognitive radios," IEEE ICASSP, Vol. 4, pp. 1357-1360, 2007. 
[16] D. Malioutov, S. Sanghavi, and A. Willsky, "Compressed sensing with sequential observations," Proc. IEEE ICASSP'08, Las Vegas, NV, USA, pp. 3357-3360, Apr. 2008.

[17] S. Bokharaiee, HN Naguyen, E Shwedyk, "Blind Spectrum Sensing for OFDM-Based Cognitive Radio Systems," IEEE Transactions on Vehicular Technology, Vol. 60, No. 3, 2011.

[18] M. Farrag, Muta, M. El-Khamy, H. Furukawa, "Wideband cooperative compressive spectrum sensing for cognitive radio systems using distributed sensing matrix" IEEE Confrence on Vehicular Technology, pp. 1-6, 2014.

[19] J Liu, S Feng, H Wang, "Comb-type pilot aided channel estimation in non-contiguous OFDM systems for cognitive radio," Proceedings 5th International Conference Wireless Communications, Networking and Mobile Computing, Beijing, China, pp. 1-4, 2009.

[20] D Hu, L He, "Pilot design for channel estimation in OFDM-based cognitive radio systems," Proc. IEEE Global Telecommunications Confrence (GLOBECOM), Miami, FL, USA, pp. 1-5, 2005

[21] $\mathrm{D} \mathrm{Hu}, \mathrm{L} \mathrm{He}, \mathrm{X}$ Wang, "An efficient pilot design method for OFDM-based cognitive radio systems," IEEE Transaction Wireless Communication, Vol. 10, No. 4, pp. 1252-1259, 2011.

[22] Z Hasan, G Bansal, E Hossain, V Bhargava, "Energy-efficient power allocation in OFDM-based cognitive radio systems: A risk-return models," IEEE Transaction on Wireless Communication, Vol. 8, No. 12, pp. 6078-6088, 2009.

[23] D. Hu, L. He, and X. Wang, "An efficient pilot design method for OFDM-based cognitive radio systems," IEEE Transaction on Wireless Communication, Vol. 10, No. 4, pp. 1252-1259, 2011.

[24] E. Manasseh, S. Ohno, and M. Nakamoto, "Pilot design for noncontiguous spectrum usage in OFDMbased cognitive radio networks," Proc. EUSIPCO, Bucharest, Romania, pp. 465-469, 2012.

[25] Min Jia, X Liu and X Gu "Channel Estimation Algorithm Based on Compressive Sensing for NCOFDM Systems in Cognitive Radio Context," International Journal of Advancements in Computing Technology, Vol. 5, No. 1, pp. 343-351, January 2013.

[26] Chenhao Qi, G Yue, L Wu and A Nallanathan, "Pilot Design for Sparse Channel Estimation in OFDM-Based Cognitive Radio Systems," IEEE Transactions on Vehicular Technology, Vol 63, No. 2, pp. 982-987, 2014.

[27] Bremen, Germany, May 11-14, 2009. R. Baraniuk. “A lecture on compressive sensing,” IEEE Signal Processing Magazine, vol. 24, no. 4, pp. 118-121, 2007.

[28] Emmanuel J. Candès, "The restricted isometry property and its implications for compressed sensing," Applied \& Computational Mathematics, California Institute of Technology, Pasadena, 2008.

[29] R. Nef, A. Zakhor. "Very low bit rate video coding based on matching pursuits," IEEE Transactions on Circuits and Systems for Video Technology, vol 7, no. 1, pp. 158-171, 1997.

[30] J. A. Tropp and A. C. Gilbert, "Signal recovery from partial information by orthogonal matching pursuit," IEEE Trans. Inform. Theory, vol 53, pp. 4655-4666, 2007.

[31] D. Needell and J. A. Tropp, "Cosamp: Iterative signal recovery from incomplete and inaccurate samples," Preprint, Mar 2008.

[32] C. Gilbert, M. J. Slrauss, J A Tropp, "Algorithmic linear dimension reduction in the norm for sparse vectors," 44th Annual Allerton Conference on Communication, Control, and Computing, 2006.

[33] T.T. Cai, Lie Wang, "Orthogonal Matching Pursuit for Sparse Signal Recovery With Noise," IEEE Transactions on Information Theory, vol. 57, no. 7, pp. 4680-4688, July 2011.

[34] C. Qi and L. Wu, “Application of compressed sensing to DRM channel estimation,” Proc. 73rd IEEE VTC-Spring, Budapest, Hungary, pp. 1-5, May 2011.

[35] W. Waigang, Z yang, B Gu, "A non-reconstruction method of compressed spectrum sensing," IEEE conference on Wireless Communications on Networking and Mobile Computing, Wuhan, pp. 1-2, 2011.

[36] L. Jia, Y. Shen, Q. weng, "Stepwise suboptimal iterative hard thresholding algorithm for compressive sensing" IEEE International Instrumentation and Measurement Technology Conference (I2MTC), Graz, pp. 1332-1336 2012

[37] X. Fengmin, W. Shanhe, X Zongben, "A hybrid hard thresholding algorithm for compressed sensing," IEEE 3rd International Conference on Communication Software and Networks (ICCSN), pp 192-196, May 2011.

[38] Hongjian Sun, Wei Yu Chiu, A Nallanathan, "Adaptive compressive spectrum sensing for wideband cognitive radio" IEEE Communication Letters, vol. 16, pp. 1812-1815, Nov 2012.

[39] T. Tony Cai, Lie Wang, “Orthogonal matching pursuit for sparse signal recovery with noise," IEEE Transactions on Information theory, vol. 57, no. 7, pp. 4680-4688, July 2011.

[40] P. Flandrin, “Time frequency/ time scale analysis," San Diago (CA), Academic Press, 1999. 
[41] G Gasso, A Rakotomamonjy and S Canu, "Solving non-convex Lasso type problems with DC programming," IEEE Workshop on Machine Learning for Signal Processing, Cancun, pp. 450455,2008 .

[42] D. Needell, J. Tropp, "Greedy signal recovery review," 42nd Asilomar Conference on Signals, Systems and Computers, CA, pp. 1048-1050, 2008.

[43] Jerome Gilles, "Empirical wavelet transform," IEEE Transactions on Signal Processing, vol 61, pp. 3999-4010, August 15, 2013.

[44] T.T. Do, Lu Gan, Nam Nguyen. "Sparsity adaptive matching pursuit algorithm for practical compressed sensing," IEEE Asilomar Conference on Signals, Systems, and Computers, Pacific Grove, California, pp. 581-587, 2008.

[45] D. Needell and J. Tropp, "CoSaMP: Iterative signal recovery from incomplete and inaccurate samples,” Appl. Comp. Harmonic Anal., vol. 26, no. 3, pp. 291-432, 2008.

\section{Authors}

Naveen K. Yadav received his B. Tech degree from Gautam Buddh Technical University in 2010 and M. Tech degree from National Institute of Technology, Jalandhar, India in 2015. He has worked as Assistant Professor in Electronics and Communication Department, J. S. Institute of Technology, Uttar Pradesh from year 2010 to 2013. Currently, he is working as Assistant Professor in Department of ECE., S.S. Institute of Technology, Aligarh. His research interests include cognitive radio, wireless sensor networks, massive MIMO and smart grid.

Neetu Sood received the B. Tech degree (with Honour) in Electronics and Communication from Sant Longowal Institute of Engineering and Technology, India 2000. In year Jan. 2002, she completed her M.Eng. Degree (With Honour) in Electronics and Communication from Thapar University, Patiala, India and PhD in Electronics and Communication in year 2013. From year 2002 to 2007, she worked with DAV Institute of Engineering and Technology, Jalandhar and Guru Nanak Dev University (Regional

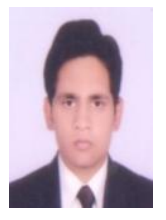
Campus), Jalandhar. Currently, she is working as Assistant Professor at Dr. B Ambedkar National Institute of Technology, Jalanadhar. Her current research includes the simulation of wireless systems based on OFDM and simulation of fading channels. 